\title{
S100A8/A9 (Calprotectin), Interleukin-6, and C-Reactive Protein in Obesity and Diabetes before and after Roux-en-Y Gastric Bypass Surgery
}

\author{
Louise Lylloffa ${ }^{a}$ Lise Bathum $^{a}$ Sten Madsbad ${ }^{b}$ \\ Josefine Liv Gilling Grundtvig ${ }^{b}$ Inge Nordgaard-Lassen ${ }^{c}$ \\ Mogens Fenger ${ }^{a}$ \\ a Department of Clinical Biochemistry, Copenhagen University Hospital Hvidovre, Hvidovre, \\ Denmark; ${ }^{b}$ Department of Endocrinology, Copenhagen University Hospital Hvidovre, \\ Hvidovre, Denmark; ${ }^{c}$ Gastrounit, Medical Division, Copenhagen University Hospital \\ Hvidovre, Hvidovre, Denmark
}

\section{Keywords}

Inflammation · Diabetes mellitus type $2 \cdot$ Obesity $\cdot$ Bariatric surgery

\begin{abstract}
Background: In obesity, which is a major contributor to insulin resistance and diabetes, the circulating level of S100A8/A9 (calprotectin) is elevated and declines after Roux-en-Y gastric bypass surgery (RYGB). However, studies on S100A8/A9 and the pathophysiological mechanisms in insulin resistance and diabetes are few and contradictory. Methods: We studied 48 subjects who underwent RYGB, comprising a non-diabetic control group and two diabetic groups in whom diabetes either regressed or persisted, 6-12 months post-surgically. S100A8/ A9, interleukin 6 (IL-6) as well as other inflammatory and diabetes-related markers were measured pre- and post-surgically. Results: Significant and similar decreases of BMI were found in all groups. S100A8/A9 and IL-6 decreased significantly in the group with diabetes remission and in the control group, but not in the group with persistent diabetes. The relative changes in S100A8/A9 and IL-6 correlated significantly $(r=0.905, p=0.005)$ only in the group with persistent diabetes. In contrast, leukocyte count and C-reactive protein correlated significantly to S100A8/A9 only in the control group. Conclusion: Our study is suggestive of S100A8/A9 and IL- 6 being related to a persistent diabetes status post-surgically and of different pathophysiological mechanisms being involved in the post-surgical changes in the three groups, despite similar decreases in BMI.

(C) 2017 The Author(s)

Published by S. Karger GmbH, Freiburg
\end{abstract}




\section{Introduction}

Obesity is a major contributor to the development of insulin resistance, which in turn may lead to type 2 diabetes mellitus (T2DM) [1]. The insulin resistance appears to be associated with low-grade chronic inflammation, originating from adipose tissue [1,2]. Obesityassociated chronic inflammation is characterized by an immense infiltration of macrophages in the adipose tissue [1] with secretion of pro-inflammatory cytokines, such as interleukin 6 (IL-6) [2, 3], and of peripheral leukocytosis [4, 5].

Leukocyte count and C-reactive protein (CRP) are increased in obesity and decline after bariatric surgery [6,7]. Further, elevated CRP is shown to be associated with metabolic syndrome [8] and the risk of development of T2DM [9]. Studies on IL-6 and T2DM have been conflicting, and the role of IL- 6 in the development of insulin resistance and T2DM is controversial [1, 9-11].

S100A8/A9, a heterodimer of the two calcium-binding proteins S100A8 and S100A9, has been found elevated in a wide variety of inflammatory conditions. The main sources of S100A8/A9 are cells of myeloid origin, primarily neutrophils, monocytes, and early stages of macrophages [12]. Furthermore, platelets contain and release S100A8/A9 [13], and expression of S100A8 and S100A9 can be induced in epithelial cells under inflammatory conditions [12]. S100A8/A9 has diverse intra- as well as extracellular functions and serves as a damage-associated molecular pattern (DAMP), being a ligand of Toll-like receptor 4 (TLR-4). Interaction with TLR-4 results in production of pro-inflammatory cytokines, neutrophil adhesion to endothelium, and recruitment of neutrophils to sites of inflammation [12].

In obese mice in which leukocytosis is also seen, a recent study by Nagareddy et al. [14] showed that S100A8/A9 and other TLR-4 ligands are upregulated in adipose tissue and interacts with TLR-4 on adipose tissue macrophages (ATMs). This interaction promotes the production of interleukin $1 \beta$ (IL-1 $\beta$ ) which in turn stimulates the bone marrow to produce monocytes and neutrophils. The monocytes infiltrate the adipose tissue, and thereby a vicious circle is induced.

In humans, plasma S100A8/A9 levels have been found elevated in obese subjects [1517]. However, in relation to insulin resistance and T2DM results are conflicting; one study did not find any correlation between circulating S100A8/A9 and markers of glucose metabolism [15], whereas other researchers have found S100A8/A9 to be correlated to insulin resistance [16] and suggestive of being involved the development of insulin resistance [17].

The aim of this study was to investigate the changes in the circulating level of S100A8/ A9 and the inflammatory markers CRP, IL-6, and leukocyte count after bariatric surgery in three phenotypically distinct groups in relation to glucose tolerance. We examined one group with diabetes both pre- and post-surgically, one group with diabetes pre-surgically but not post-surgically, and one group without diabetes before as well as after surgery. By choosing this approach, we attempt to distinguish between diabetes and obesity-related contributions to the inflammatory status.

\section{Material and Methods}

\section{Subjects}

This study included subjects selected among the participants of the AdiKir Study Cohort, Hvidovre Hospital, Copenhagen [18]. In brief, subjects suffering from severe obesity who underwent RYGB surgery were monitored clinically and para-clinically as well as pre- and post-surgically.

All participants had given written informed consent before participation, and the study was approved by the Scientific Ethics Committee of Capital Region of Denmark (HD-2009-78). 
Lylloff et al.: S100A8/A9 (Calprotectin), Interleukin-6, and C-Reactive Protein in Obesity and Diabetes before and after Roux-en-Y Gastric Bypass Surgery

The selection was made among participants with leukocyte and platelet counts available. Further, in order to explore biochemically changes related to diabetes, the selection was based on diabetes status preand post-surgically. Subjects with an $\mathrm{HbA1C} \geq 48 \mathrm{mmol} / \mathrm{mol}$ or patient record information on antidiabetic therapy were defined as diabetics.

In total, 48 participants, comprising three subgroups according to diabetes status, were selected. The groups were named and composed as follows: T2DM-/-, a control group including 19 subjects with normal glucose tolerance pre- as well as post-surgically; T2DM+/-, comprising 19 diabetic subjects in whom diabetes remitted post-surgically; and T2DM+/+, consisting of 10 subjects who were diabetic prior to surgery and remained diabetic post-surgically.

For each participant, anthropometric data and blood samples from two visits, one pre-surgically and one 612 months post-surgically, were chosen.

\section{Sample Collection, Preparation and Storage}

All samples were collected after an overnight fast. Plasma samples used for the analyses of S100A8/A9 and IL-6 were collected in EDTA tubes and kept on ice until centrifugation, and serum was collected for the measurement of C-peptide and insulin. Both specimens were centrifuged at 2,500 $\times \mathrm{g}$ at $4{ }^{\circ} \mathrm{C}$ for $10 \mathrm{~min}$ and aliquoted into cryo tubes. All samples were processed within $2 \mathrm{~h}$ and kept at $-20^{\circ} \mathrm{C}$ for maximum 1 week before storage at $-80^{\circ} \mathrm{C}$. During the same venipuncture, lithium-heparin plasma and EDTA blood were collected for analysis of CRP and leukocyte count, respectively. Finally, a venous and/or and capillary sample was collected for immediate measurement of glucose and HbA1C.

\section{Anthropometric Data}

At each clinical visit the participants were weighed, and information on antidiabetic medication was collected. The height was measured at the first clinical visit.

\section{Biochemical Measurements}

S100A8/A9 and IL-6 values were measured by use of enzyme-linked immuno-sorbent assays (ELISA). Samples from each of the three groups were run on separate plates with separate standard curves. Pre- and post-surgical samples from each participant were placed consecutively on each plate, and all samples were measured in duplicate. For each of the two analyses, assay kits of the same lot were used for all samples.

S100A8/A9 was measured using the 'Bühlmann MRP8/14 ELISA' assay from Bühlmann Laboratories AG (Schönenbuch, Switzerland) with a limit of detection (LOD) of $400 \mu \mathrm{g} / \mathrm{l}$ and an intra- and inter-assay coefficient of variation (CV) of $4.6 \%$ and $6.9 \%$ (at 1,400 $\mathrm{g} / \mathrm{l}$ ), respectively. Duplicate measurements differing more than 20\% were excluded. 19 (4 pre-surgically and 15 post-surgically) of the 96 measurements of P-S100A8/A9 were below LOD. In order not to lose information, we chose to assign all S100A8/A9 measurements below LOD a value of $400 \mu \mathrm{g} / \mathrm{l}$.

IL-6 was measured using the 'Human IL-6 High Sensitivity ELISA' assay from eBioscience Inc. (San Diego, CA, USA) with a LOD of $0.03 \mathrm{pg} / \mathrm{l}$ and intra-assay and inter-assay CV of $5.2 \%$ and $7.3 \%$, respectively. Duplicate measurements differing more than $12 \%$ were excluded.

C-peptide and insulin were analyzed on Immulite ${ }^{\circledR} 2000$ (Siemens Healthcare Diagnostics, Gwynedd, UK) with lowest reportable ranges of $33 \mathrm{pmol} / \mathrm{l}$ and $14.4 \mathrm{pmol} / \mathrm{l}$, intra-assay $\mathrm{CV}$ of $2.3 \%$ and $5.5 \%$, and interassay CV of $2.9 \%$ and $7.3 \%$, respectively $[19,20]$.

CRP was analyzed on a Cobas $6000^{\circledR}$, C501 (Roche Diagnostics, Rotkreutz, Switzerland) with a LOD of $0.3 \mathrm{mg} / \mathrm{l}$.

CRP values of 16 ( 1 pre-surgically and 15 post-surgically) measurements were at or below LOD, and these were all assigned a value of $0.3 \mathrm{mg} / \mathrm{l}$.

Leukocyte count was performed on a Sysmex XE-5000 ${ }^{\mathrm{TM}}$ (Sysmex Coorporation, Kobe, Japan). Glucose measurements were carried out on HemoCue ${ }^{\circledR}$ Glucose 201+ (HemoCue AB, Ängelholm, Sweden) and reported as plasma equivalent values. HbA1C was analyzed on a Tosoh G7 (Tosoh Europe N.V, Tessenderlo, Belgium).

Homeostatic model assessment of insulin resistance (HOMA-IR) was calculated as: (fasting serum insulin $(\mathrm{mU} / \mathrm{l}) \times$ fasting plasma glucose $(\mathrm{mmol} / \mathrm{l})) / 22.5$ [21]. HOMA-IR is only reliable to use in T2DM not treated with insulin [21]. 
Lylloff et al.: S100A8/A9 (Calprotectin), Interleukin-6, and C-Reactive Protein in Obesity and Diabetes before and after Roux-en-Y Gastric Bypass Surgery

Table 1. Pre- and post-surgical median levels of BMI, HOMA-IR and diabetes and inflammatory variables of the three groups

\begin{tabular}{|c|c|c|c|c|c|c|c|}
\hline \multirow[t]{2}{*}{ Group } & \multicolumn{2}{|c|}{$\mathrm{T} 2 \mathrm{DM}+/+$} & \multicolumn{2}{|c|}{$\mathrm{T} 2 \mathrm{DM}+/-$} & \multicolumn{2}{|c|}{ T2DM-/- } & \multirow[t]{2}{*}{$\mathrm{p}$ value } \\
\hline & $\mathrm{n}$ & median (95\% CI levels) & $\mathrm{n}$ & median (95\% CI levels) & $\mathrm{n}$ & median (95\% CI levels) & \\
\hline \multicolumn{8}{|l|}{ Pre-surgically } \\
\hline BMI, $\mathrm{kg} / \mathrm{m}^{2}$ & 10 & $42.3(38.8-45.8)$ & 19 & $42.9(40.8-45.8)$ & 19 & $42.3(37.3-46.2)$ & 0.645 \\
\hline $\mathrm{HbA} 1 \mathrm{C}, \mathrm{mmol} / \mathrm{mol}$ & 9 & $\left.56(49-86) a)^{* * * *} c\right)^{*}$ & 19 & $45(41-50) \mathrm{b})^{* * * *}$ & 19 & $37(35-40)$ & $<0.0001$ \\
\hline Glucose, mmol/l & 9 & $\left.8.6(7.2-19.0) \mathrm{a})^{* * * * *} \mathrm{c}\right)^{* *}$ & 17 & $6.3(5.6-7.4) \mathrm{b})^{*}$ & 19 & $5.6(5.1-6.0)$ & 0.0002 \\
\hline C-peptide, pmol/l & 10 & $\left.843(655-1,480) a)^{\text {ns }} c\right)^{n s}$ & 19 & $1,258(947-1,635) b)^{* *}$ & 19 & $808(682-1,291)$ & 0.025 \\
\hline Insulin, $\mathrm{pmol} / \mathrm{l}$ & 4 & $117(70-163)$ & 18 & 145 (119-198) & 19 & $105(64-165)$ & nd \\
\hline HOMA-IR & 4 & $6.75(3.70-15.81)$ & 16 & $6.28(4.15-8.81)$ & 19 & $3.76(2.27-6.32)$ & nd \\
\hline S100A8/A9, $\mu \mathrm{g} / \mathrm{l}$ & 10 & $1,248(701-1,972)$ & 19 & $979(649-1,195)$ & 18 & $853(630-1,204)$ & 0.278 \\
\hline IL-6, ng/l & 9 & $2.3(1.5-3.9)$ & 17 & $2.4(2.0-3.4)$ & 18 & $2.6(2.1-3.4)$ & 0.707 \\
\hline $\mathrm{CRP}, \mathrm{mg} / \mathrm{l}$ & 9 & $3.0(1.0-9.0)$ & 19 & $5.0(1.0-8.0)$ & 19 & $6.0(3.0-8.0)$ & 0.642 \\
\hline Leukocyte count $\times 10^{9} / 1$ & 10 & $7.0(4.5-11.6)$ & 19 & $6.5(5.8-7.2))$ & 19 & $6.0(5.3-7.7)$ & 0.456 \\
\hline \multicolumn{8}{|l|}{ Post-surgically } \\
\hline BMI, $\mathrm{kg} / \mathrm{m}^{2}$ & 10 & $31.0(27.6-34.6)$ & 19 & $30.5(29.2-33.6)$ & 19 & $30.1(26.8-31.7)$ & 0.492 \\
\hline $\mathrm{HbA} 1 \mathrm{C}, \mathrm{mmol} / \mathrm{mol}$ & 10 & $\left.48(42-51) a)^{* * * *} c\right)^{* * * *}$ & 19 & $37(33-38) b)^{*}$ & 19 & $34(32-36)$ & $<0.0001$ \\
\hline Glucose, mmol/l & 10 & $\left.7.2(5.5-9.5) \mathrm{a})^{* * *} \mathrm{c}\right)^{* *}$ & 18 & $5.2(5.0-5.6) b)^{\mathrm{ns}}$ & 19 & $5.0(4.9-5.3)$ & 0.0009 \\
\hline C-peptide, pmol/l & 10 & $634(473-798)$ & 19 & $738(516-847)$ & 19 & $523(417-645)$ & 0.052 \\
\hline Insulin, pmol/l & 9 & $43(32-44)$ & 19 & $53(32-71)$ & 17 & $43(31-64)$ & 0.317 \\
\hline HOMA-IR & 9 & $1.70(1.36-2.53)$ & 18 & $1.76(1.09-2.68)$ & 17 & $1.33(0.98-2.08)$ & 0.312 \\
\hline S100A8/A9, $\mu \mathrm{g} / \mathrm{l}$ & 10 & $607(400-863)$ & 19 & $484(400-710)$ & 18 & $537(400-691)$ & 0.585 \\
\hline IL-6, ng/l & 8 & $1.2(0.8-5.0)$ & 17 & $1.7(1.2-2.4)$ & 17 & $1.7(1.2-2.9)$ & 0.525 \\
\hline $\mathrm{CRP}, \mathrm{mg} / \mathrm{l}$ & 10 & $\left.0.7(0.3-3.0) a)^{\mathrm{ns}} c\right)^{\mathrm{ns}}$ & 19 & $1.0(0.3-1.0) b)^{*}$ & 19 & $2.0(1.0-4.0)$ & 0.042 \\
\hline Leukocyte count $\times 10^{9} / \mathrm{l}$ & 10 & $6.3(5.3-7.0)$ & 19 & $6.0(5.2-6.4)$ & 19 & $5.6(4.8-6.4)$ & 0.497 \\
\hline
\end{tabular}

a) Significance levels of differences between T2DM+/+ and T2DM-/-.

b) Significance levels of differences between T2DM+/- and T2DM-/-.

c) Significance levels of differences betweenT2DM+/+ and T2DM+/-.

${ }^{*} \mathrm{p} \leq 0.05 ;{ }^{* *} \mathrm{p} \leq 0.01 ;^{* * *} \mathrm{p} \leq 0.001 ;{ }^{* * * *} \mathrm{p} \leq 0.0001 ; \mathrm{ns}=$ no significance; $\mathrm{nd}=$ not determined.

Statistics

Statistical analyses and graphics were performed with GraphPad Prism, Version 7.02 (GraphPad Software, San Diego, CA, USA). Data were tested for normality (Shapiro-Wilk Test). As most data were not normally distributed and due to the sample sizes, non-parametric statistics were chosen for all variables.

The differences between pre- and post-surgical levels were assessed by Wilcoxon matched-pairs signed ranks test and Mann-Whitney test for paired and unpaired data. Comparisons of data between the three groups were made by Kruskal-Wallis Test. The correlations between variables were evaluated by Spearman rank test.

A p value of less than 0.05 was considered significant and all $p$ values are two-sided.

In order to remove between-subject variation, relative decreases of the single variables were calculated as: (pre-surgical value - post-surgical value) / pre-surgical value.

Due to missing data, the number of results included in each of the single analyses varies. The number of results is given in each table.

\section{Results}

Participant Characteristics and Study Visits

48 participants of both genders ( 31 females) with a median age of 49.7 ( $95 \%$ confidence interval (CI) of the median 46.1-52.3) years, at the pre-surgical blood sampling were included. 
Median time from the pre-surgical clinical visit and pre-surgical blood sampling to surgery were 97.5 (63-127) and 110.5 (84-137) days, respectively. Median time from surgery to the post-surgical clinical visit and post-surgical blood sampling were $207.0(190-344)$ and 193.0 (182-342) days, respectively.

Median pre- and post-surgical BMI of all participants were $42.3(40.8-44.5)$ and 30.5 (29.4-31.7), $\mathrm{kg} / \mathrm{m}^{2}$, respectively, and differed significantly $(\mathrm{p}<0.0001)$.

Pharmacological anti-diabetic treatment included mono- or poly-therapy of metformin, sulunylurea, insulin analogues, glucagon-like petide- 1 agonist and/or dipeptidyl peptidase- 4 inhibitors. Insulin analogues were administered pre-surgically in 7 post-surgically in 1 participant, and samples were excluded for data analysis of insulin and HOMA-IR.

The median pre- and post-surgical levels and relative changes of diabetes and inflammatory variables of all participants are shown in supplementary table 1 (available at $h t t p: / /$ content.karger.com/ProdukteDB/produkte.asp?doi=478097).

Pre- and Post-Surgical Levels of BMI and Biochemical Variables

Pre- and post-surgical median levels of BMI and diabetes as well as inflammatory variables of the three groups are shown in table 1.

The median BMI did not differ between the three groups, neither pre- nor post-surgically. As expected, pre- as well as post-surgical $\mathrm{HbA1C}$ and plasma glucose levels differed significantly between the three groups. Pre-surgical C-peptide levels differed significantly, while no difference was found post-surgically. Comparison of pre-surgical insulin and HOMA-IR levels of all groups were not done, due to the sample sizes. Post-surgically, no differences were found between the three groups.

Pre-surgical CRP levels did not differ, whereas post-surgical levels differed significantly between the three groups. None of the other inflammatory markers showed any differences between the three groups, neither pre- nor post-surgically.

HbA1C levels differed significantly between each of the groups, pre- as well as post-surgically. Pre-surgical plasma glucose levels differed significantly between each of the three groups, while the post-surgical plasma glucose level of T2DM+/+ was significantly higher than that of T2DM+/- and T2DM-/- and no difference was found between T2DM+/- and T2DM-/-. The pre-surgical C-peptide levels was significantly higher in T2DM+/- compared to T2DM-/-, whereas no differences were found between $\mathrm{T} 2 \mathrm{DM}+/+$ and $\mathrm{T} 2 \mathrm{DM}-/-$ or $\mathrm{T} 2 \mathrm{DM}+/+$ and $\mathrm{T} 2 \mathrm{DM}+/-$. The post-surgical CRP level was significantly higher in T2DM-/compared to T2DM+/-, while no significant differences were found between TD2DM+/+ and the other two groups.

\section{Absolute Changes}

HbA1C, plasma glucose, C-peptide, S100A8/A9, and CRP decreased significantly in all three groups as shown in figure $1 \mathrm{a}$, b. Insulin and HOMA-IR decreased significantly in T2DM+/and T2DM-/-. As only 4 participants in T2DM+/+ were not treated with insulin, no comparison between pre- and post-surgical levels of insulin and HOMA-IR was performed in this group. IL-6 decreased significantly in T2DM+/-, while no significant changes were seen in T2DM-/or T2DM+/+. Significant decreases of leukocyte counts were found in T2DM-/- and T2DM+/-, but not in $\mathrm{T} 2 \mathrm{DM}+/+$.

\section{Relative Changes}

In order to remove between-subject variation and thus make results comparable, the relative changes of each of the variables were calculated (supplementary table 2, available at http://content.karger.com/ProdukteDB/produkte.asp?doi=478097). As shown in figure 2a, the relative decrease of $\mathrm{HbA} 1 \mathrm{C}$ was significant in all groups and differed significantly between 
a
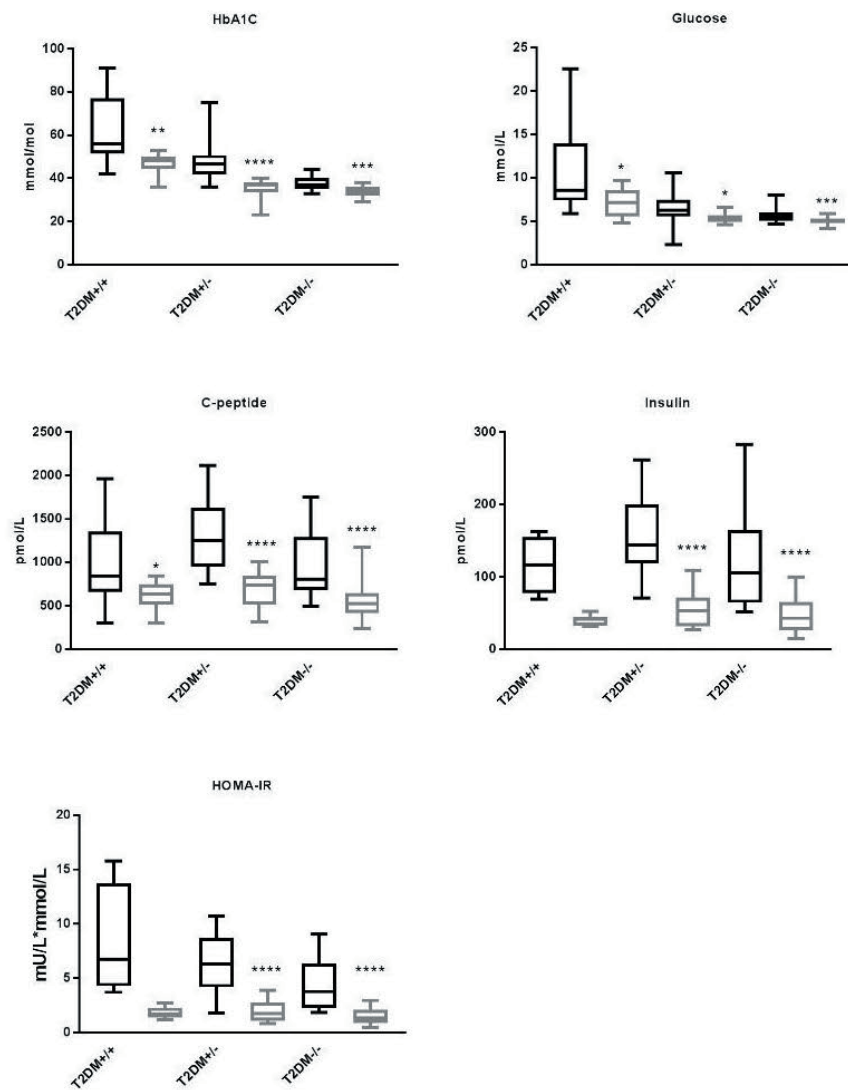

b
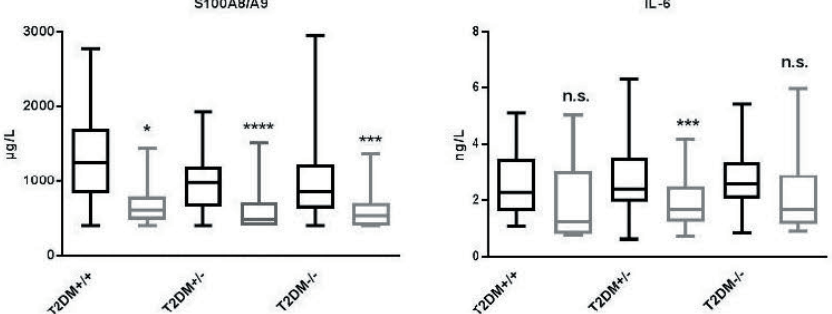
post-surgically (grey). Boxes represent interquartile ranges and whiskers minimum and maximum values. Due to sample sizes comparisons of pre- and post-surgical levels of insulin and HOMAIR of T2DM+/+ were not done. Significance levels of the differences between pre- and post-surgical levels are indicated by * $\mathrm{p} \leq$ $0.05,{ }^{* *} \mathrm{p} \leq 0.01,{ }^{* * *} \mathrm{p} \leq 0.001$ and $* * * * \mathrm{p} \leq 0.0001$, n.s. $=$ no significance.
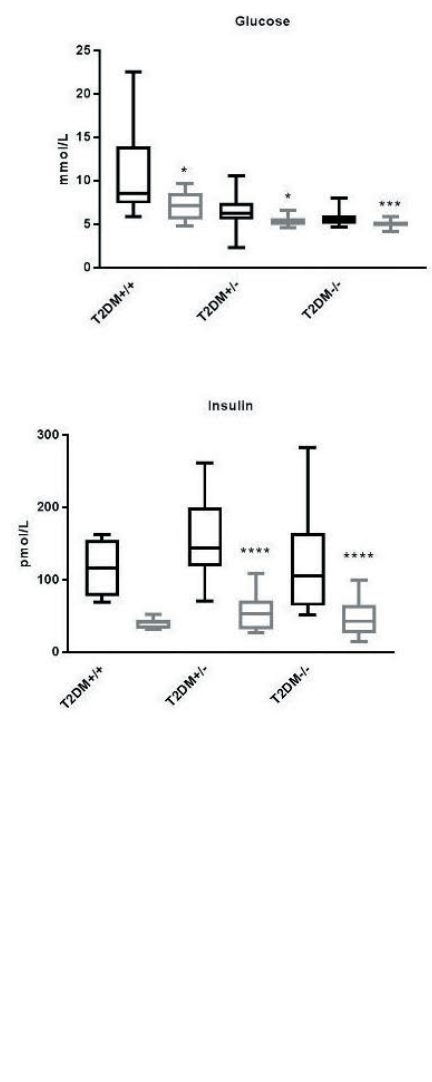

Fig. 1. Pre- and post-surgical median levels of diabetes and inflammatory variables. Median levels of diabetes (a) and inflammatory (b) variables pre- (black) and 
Lylloff et al.: S100A8/A9 (Calprotectin), Interleukin-6, and C-Reactive Protein in Obesity and Diabetes before and after Roux-en-Y Gastric Bypass Surgery

a
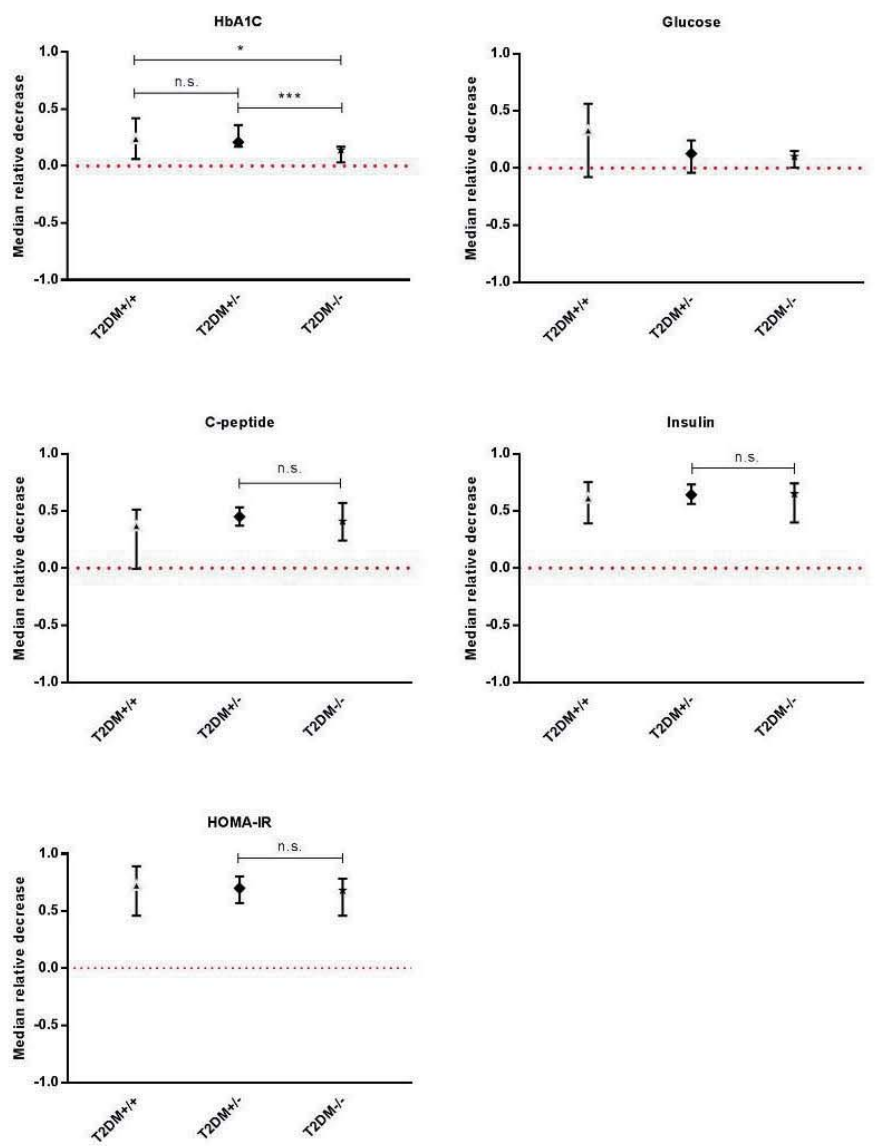

Fig. 2. Relative changes in diabetes and inflammatory variables. Median levels of relative changes in diabetes (a) and inflammatory (b) variables. Whiskers represent 95\% CIs of the medians. As S100A8/A9 and CRP values were truncated at values of $400 \mu \mathrm{g} / \mathrm{l}$ and $0.3 \mathrm{mg} / \mathrm{l}$, respectively, the decreases of these variables are minimum decreases. Due to sample sizes comparisons to insulin and HOMA-IR of T2DM+/+ were not done. Significance levels of differences of the relative decreases between the single groups are indicated by *p $\leq 0.05, * \mathrm{p} \leq$ $0.01,{ }^{* * *} \mathrm{p} \leq 0.001$ and ${ }^{* * * *} \mathrm{p} \leq$ 0.0001, n.s. $=$ no significance.
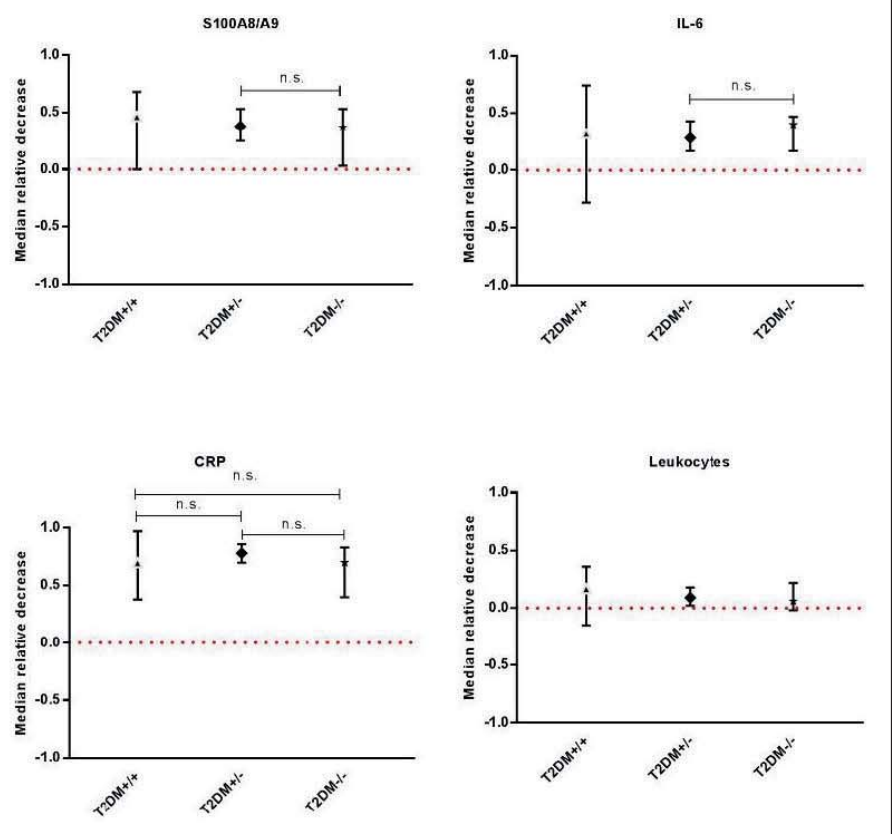
Table 2. Spearman rank correlation coefficients of the relative decreases of S100A8/A9 to those of BMI, HOMA-IR and diabetes and inflammatory variables

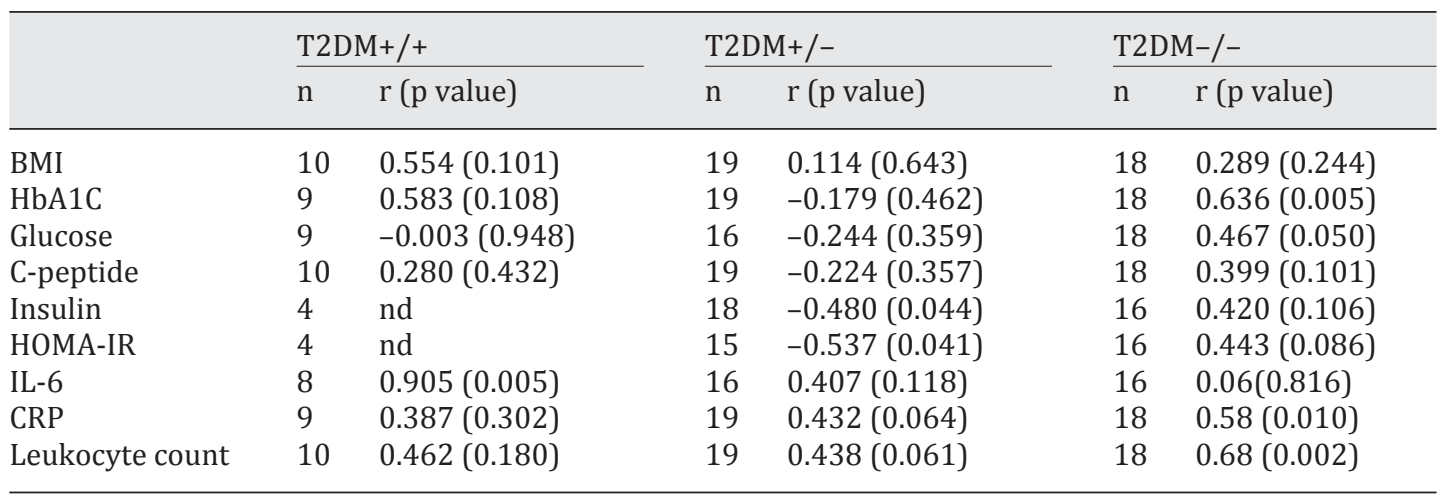

Spearman rank correlations coefficients $r$ ( $p$ value) of the relative decreases of S100A8/A9 to those of BMI, HOMA-IR and diabetes and inflammatory variables. Due to the sample sizes the correlations to insulin and HOMA-IR in T2DM+/+ were not done (nd). Significant correlations are shown in italics.

each of the groups. The relative decrease of plasma glucose did not reach significance in any of the groups. C-peptide decreased significantly in T2DM-/- and T2DM+/-, but not in $\mathrm{T} 2 \mathrm{DM}+/+$, and did not differ between T2DM-/- and T2DM+/-. Insulin decreased significantly in T2DM+/- and T2DM-/-, but the difference between the two groups was not significant.

Significant decreases of S100A8/A9 and IL-6 were found in group T2DM+/- and T2DM-/-, but not in T2DM+/+ (fig. 2b). The decreases of S100A8/A9 and IL-6 did not differ between T2DM+/- and T2DM-/-. CRP decreased significantly in all three groups, but no differences were found between any of the groups. A significant decrease in leukocyte count was only found in T2DM+/-.

The correlations of the relative decreases in S100A8/A9 and those of BMI and diabetes and inflammatory variables are shown in table 2.

The relative decrease of S100A8/A9 was not correlated to BMI in any of the groups. A strong correlation between the relative decreases of S100A8/A9 and that of IL- 6 in T2DM+/+, but not in T2DM+/- and T2DM-/-, was found. Interestingly, the correlation of the decrease in S100A8/A9 and that of CRP and leukocyte count progressed across the three groups being non-significant in T2DM+/+ and moderately to highly significant in group T2DM-/-. The relative decreases of IL- 6 did not correlate significantly to neither inflammatory or diabetes variables, nor to BMI, in any of the groups (supplementary table 3, available at $h t t p: / /$ content. karger.com/ProdukteDB/produkte.asp?doi=478097).

\section{Discussion}

In this study we explored changes in S100A8/A9 and other inflammatory markers after bariatric surgery in two phenotypically distinct diabetes groups compared to a non-diabetic control group before and after surgery.

We found significant absolute decreases of S100A8/A9 and CRP in each of the groups, which is in accordance with previous studies on S100A8/A9, CRP, and weight loss $[6,16,17$, 22-24] and might reflect an overall decrease of the chronic low-grade inflammation seen in obesity. However, pre- and post-surgical levels showed rather large 95\% CIs of all variables, especially those of the T2DM+/+ group. Therefore, we chose to focus on the relative changes. 
Our study showed that the relative decreases of S100A8/A9 and IL-6 were significant in $\mathrm{T} 2 \mathrm{DM}-/-$ and $\mathrm{T} 2 \mathrm{DM}+/-$, but not in $\mathrm{T} 2 \mathrm{DM}+/+$. Further, a significant correlation between the decreases of S100A8/A9 and IL-6 in T2DM+/+ was seen. This was, despite similar weight loss, not the case in $\mathrm{T} 2 \mathrm{DM}+/-$ and $\mathrm{T} 2 \mathrm{DM}-/-$, suggesting that a pathophysiological mechanism involving S100A8/A9 and IL-6 is related to a persistent diabetic status. The decreases of S100A8/A9 and CRP did not correlate in T2DM+/+, while in T2DM-/- significant correlations were found. The same trend was observed for changes in leukocyte count. Thus, these patterns of changes in inflammatory markers strongly indicate that the three groups are governed by different pathophysiological mechanisms.

After surgery, improvements in both hepatic and peripheral insulin resistance have been reported [25]. In the present study a marked improvement in insulin resistance was observed after surgery in $\mathrm{T} 2 \mathrm{DM}+/-$ and $\mathrm{T} 2 \mathrm{DM}-/-$. In $\mathrm{T} 2 \mathrm{DM}+/-$, but not in $\mathrm{T} 2 \mathrm{DM}-/-$, a significant correlation between the relative decrease of S100A8/A9 and relative changes of HOMA-IR was observed. In T2DM+/+, the pre-surgical HOMA-IR could only be estimated in 4 participants not treated with insulin, but in this 4 participants the improvement in HOMA-IR seems to be similar to the changes in T2DM+/- and T2DM-/-. Taken all together, this might indicate that the changes in S100A8/A9 are not explained by improvement in insulin resistance alone, but are also related to diabetic state. Accordingly, the HOMA-IR did not differ between the three groups after surgery.

Studies in mice and humans have shown increased expression of S100A8 and S100A9 in adipose tissue of obese and diabetic subjects [14,17], and the findings of Nagareddy et al. [14] indicated that in mice S100A8/A9 is involved in a feed-forward loop that maintain a chronic inflammation of the adipose tissue. Our findings of significant post-surgical decreases in S100A8/A9 and correlations to leukocyte count and CRP in the control group are suggestive of an interruption of such a feed-forward loop. However, it does not explain the non-significant decreases of S100A8/A9 in T2DM+/+, in which the decrease of BMI did not differ from that of the two other groups. A previous study of Nagareddy et al. [26] using a type 1 diabetic mice model, suggested that hyperglycemia promotes a S100A8/A9-driven myeolopoiesis. Taken this finding into account, the non-significant decreases of S100A8/A9 and leukocyte count in the diabetes-persistent group might actually be related to a persistent hyperglycemic state. This hypothesis is supported by the finding of Nakatsuji et al. [27] that the circulating level of S100A8/A9 in T2DM patients with abdominal obesity were reduced after 3-month treatment with pioglitazone, which improves insulin sensitivity, without changes in BMI.

We did not find any correlation between the relative decreases of BMI and that of S100A8/ A9, further suggesting that changes in S100A8/A9 are not related to the weight loos induced by Roux-en-Y gastric bypass. This finding is in agreement with Nijhuis et al. [22] who compared the decreases of S100A8/A9 and BMI. Ortega et al. [16] and Catalan et al. [17] have, on the other hand, detected weak to moderate correlations of circulating levels of S00A8/A9 and BMI. Thus, whether the decrease in S100A8/A9 is related to long-term changes in body composition or to immediate changes in anatomy or inflammatory or diabetes status is not clear.

In conclusion, our study is suggestive of S100A8/A9 and IL- 6 being related to persistent diabetes post-surgically and of different pathophysiological mechanisms being involved in the three phenotypically different diabetes groups.

\section{Disclosure Statement}

The authors declare no conflict of interest. 
\begin{tabular}{l|l}
\hline DOI: $10.1159 / 000478097$ & (c) 2017 The Author(s). Published by S. Karger GmbH, Freiburg
\end{tabular} www.karger.com/ofa

Lylloff et al.: S100A8/A9 (Calprotectin), Interleukin-6, and C-Reactive Protein in Obesity and Diabetes before and after Roux-en-Y Gastric Bypass Surgery

\section{References}

1 Lackey DE, Olefsky JM: Regulation of metabolism by the innate immune system. Nat Rev Endocrinol 2016;12: 15-28.

2 Kohlgruber A, Lynch L: Adipose tissue inflammation in the pathogenesis of type 2 diabetes. Curr Diab Rep 2015;15:92.

3 Eder K, Baffy N, Falus A, Fulop AK: The major inflammatory mediator interleukin- 6 and obesity. Inflamm Res 2009;58:727-736.

4 Herishanu Y, Rogowski O, Polliack A, Marilus R: Leukocytosis in obese individuals: possible link in patients with unexplained persistent neutrophilia. Eur J Haematol 2006;76:516-520.

5 Yoshimura A, Ohnishi S, Orito C, Kawahara Y, Takasaki H, et al.: Association of peripheral total and differential leukocyte counts with obesity-related complications in young adults. Obes Facts 2015;8:1-16.

6 Chen SB, Lee YC, Ser KH, Chen JC, Chen SC, et al: Serum C-reactive protein and white blood cell count in morbidly obese surgical patients. Obes Surg 2009;19:461-466.

7 Cunha FM, Saavedra A, Barbosa J, Freitas P, Carvalho D, Varela A.: Effect of different bariatric surgery type on the leukocyte formula. Surg Obes Relat Dis 2016;12:1418-1423.

8 Mirhafez SR, Ebrahimi M, Saberi Karimian M, Avan A, Tayefi M, et al: Serum high-sensitivity C-reactive protein as a biomarker in patients with metabolic syndrome: evidence-based study with 7284 subjects. Eur J Clin Nutr 2016;70:1298-1304.

9 Wang X, Bao W, Liu J, Ouyang YY, Wang D, et al: Inflammatory markers and risk of type 2 diabetes: a systematic review and meta-analysis. Diabetes Care 2013;36:166-175.

10 Pal M, Febbraio MA, Whitham M: From cytokine to myokine: the emerging role of interleukin-6 in metabolic regulation. Immunol Cell Biol 2014;92:331-339.

11 McNelis JC, Olefsky JM: Macrophages, immunity, and metabolic disease. Immunity 2014;41:36-48.

12 Pruenster M, Vogl T, Roth J, Sperandio M: S100A8/A9: from basic science to clinical application. Pharmacol Ther 2016;167:120-131.

13 Lood C, Tyden H, Gullstrand B, Jonsen A, Kallberg E, et al.: Platelet-derived S100A8/A9 and cardiovascular disease in systemic lupus erythematosus. Arthritis Rheumatol 2016;68:1970-1980.

14 Nagareddy PR, Kraakman M, Masters SL, Stirzaker RA, Gorman DJ, et al: Adipose tissue macrophages promote myelopoiesis and monocytosis in obesity. Cell Metab 2014;19:821-835.

15 Mortensen OH, Nielsen AR, Erikstrup C, Plomgaard P, Fischer CP, et al: Calprotectin-a novel marker of obesity. PLoS One 2009;4:e7419.

16 Ortega FJ, Sabater M, Moreno-Navarrete JM, Pueyo N, Botas P, et al: Serum and urinary concentrations of calprotectin as markers of insulin resistance and type 2 diabetes. Eur J Endocrinol 2012;167:569-578.

17 Catalan V, Gomez-Ambrosi J, Rodriguez A, Ramirez B, Rotellar F, et al: Increased levels of calprotectin in obesity are related to macrophage content: impact on inflammation and effect of weight loss. Mol Med 2011; 17:1157-1167.

18 Fenger M, Hansen DL, Worm D, Hvolris L, Kristiansen VB, et al: Gastric bypass surgery reveals independency of obesity and diabetes mellitus type 2. BMC Endocr Disord 2016;16:59.

19 Siemens Healthcare Diagnostics: Package Insert, C-Peptide, for Use on IMMULITE 2000 Systems. July 29, 2008

20 Siemens Healthcare Diagnostics: Package Insert, Insulin, for use on Immulite 2000 systems. July 16, 2015.

21 Wallace TM, Levy JC, Matthews DR: Use and abuse of HOMA modeling. Diabetes Care 2004;27:1487-1495.

22 Nijhuis J, Rensen SS, Slaats Y, van Dielen FM, Buurman WA, Greve JW: Neutrophil activation in morbid obesity, chronic activation of acute inflammation. Obesity (Silver Spring) 2009;17:2014-2018.

23 Netto BD, Bettini SC, Clemente AP, Ferreira JP, Boritza K, et al: Roux-en-Y gastric bypass decreases pro-inflammatory and thrombotic biomarkers in individuals with extreme obesity. Obes Surg 2015;25:1010-1018.

24 Miller GD, Nicklas BJ, Fernandez A: Serial changes in inflammatory biomarkers after Roux-en-Y gastric bypass surgery. Surg Obes Relat Dis 2011;7:618-624.

25 Bojsen-Moller KN, Dirksen C, Jorgensen NB, Jacobsen SH, Serup AK, et al: Early enhancements of hepatic and later of peripheral insulin sensitivity combined with increased postprandial insulin secretion contribute to improved glycemic control after Roux-en-Y gastric bypass. Diabetes 2014;63:1725-1737.

26 Nagareddy PR, Murphy AJ, Stirzaker RA, Hu Y, Yu S, et al: Hyperglycemia promotes myelopoiesis and impairs the resolution of atherosclerosis. Cell Metab 2013;17:695-708.

27 Nakatsuji H, Kishida K, Funahashi T, Shimomura I: Three-month treatment with pioglitazone reduces circulating levels of S100A8/A9 (MRP8/14) complex, a biomarker of inflammation, without changes in body mass index, in type 2 diabetics with abdominal obesity. Diabetes Res Clin Pract 2012;95:e58-60. 